\title{
CONTROLE ESTATÍSTICO DE PROCESSO NO RESFRIAMENTO DE AVES: UM ESTUDO DE CASO
}

\author{
Eric Batista FERREIRA ${ }^{1}$ \\ Leonardo ELISEI JUNIOR ${ }^{2}$ \\ Matheus Vilas Boas MILITANI ${ }^{3}$
}

\begin{abstract}
${ }^{1}$ Professor Adjunto II, Instituto de Ciências Exatas, Universidade Federal de Alfenas (Unifal-MG). E-mail: eric.ferreira@unifal-mg.edu.br

${ }^{2}$ Graduando em Geografia Licenciatura e Bacharelado, Unifal-MG.

${ }^{3}$ Graduando em Ciências Biológicas Licenciatura, Unifal-MG.
\end{abstract}

RESUMO: O Controle Estatístico de Processo é um conjunto de técnicas que auxiliam na busca e manutenção da qualidade em processos produtivos. Suas principais ferramentas são as cartas de controle e os índices de capacidade do processo. $\mathrm{Na}$ cadeia produtiva de aves, a temperatura deve ser monitorada e mantida sob controle para que o produto não se deteriore e a legislação não seja desrespeitada. O presente trabalho mostra como essas ferramentas puderam ser utilizadas para detectar causas especiais de variação em uma agroindústria.

Palavras chave: Capacidade do processo, resfriamento de frangos, CEP.

\section{STATISTICAL PROCESS CONTROL FOR CHICKEN COOLING: A CASE STUDY}

\begin{abstract}
Statistical Process Control is a set of techniques that assist in finding and maintaining quality in production processes. Its main tools are control charts and process capability indices. In poultry production chain, the temperature must be monitored and kept under control so that the product does not deteriorate and the law is not flouted. This paper shows how these tools could be used to detect special causes of variation in an poutry industry.
\end{abstract}

Keywords: Process capability, poultry cooling, SPC.

\section{INTRODUÇÃO}

O desenvolvimento e aperfeiçoamento dos processos produtivos se tornam essenciais na luta pela sobrevivência das organizações. Redução de custos, melhorias dos sistemas de gestão da qualidade e aperfeiçoamento dos produtos tem estado entre as principais metas das organizações. Segundo Silva (1999) a ênfase para buscar melhorias da qualidade deve ser concentrada em melhoramentos contínuos, atitudes que, promovidas continuamente, permitam reconhecer os problemas, priorizar ações corretivas, implantá-las e dar sequência à postura próativa, agindo corretamente. Segundo Juran e Gryna (1993), algo fundamental para a 
obtenção dessas vantagens competitivas é a melhoria dos processos produtivos.

Para Borges (2009), o mundo vive um período em que o método estatístico é de suma importância e de modo progressivo vem sendo utilizado nos mais diversos campos de aplicação: seja na indústria, na agricultura, nas pesquisas genéticas e em todos os ramos do conhecimento humano.

De acordo com Thompson e Koronacki (1993), a concepção comum sobre controle estatístico é que ele alcançado por uma grande investigação, uma boa atitude e trabalho duro. Entretanto, ainda existem muitas companhias onde os empregados exibem todos esses atributos, mas a qualidade do produto é pobre. Por esse motivo, o monitoramento contínuo da variabilidade dos processos deve estar em sintonia com os modernos sistemas de qualidade e com o novo modelo de boas práticas de fabricação, particularmente na indústria alimentícia.

Um método eficiente, o Controle Estatístico de Processo (CEP), possuindo sete ferramentas que se complementam, quais sejam: estratificação, folha de verificação, gráfico de Pareto, diagrama de causa e efeito, diagrama de dispersão, histograma e gráficos de controle. O CEP é uma metodologia eficiente para estabilização, desenvolvimento e conhecimento aprofundado do processo produtivo. Para as organizações que têm conhecimento e habilidade no uso de suas ferramentas, o CEP é uma vantagem competitiva para o seu melhor desempenho. (PARANTHAMAN, 1990)

De acordo com Alencar (2004) e Cortivo (2005), com tais ferramentas, consegue-se uma descrição detalhada do comportamento do processo, identificando sua variabilidade e possibilitando seu controle ao longo do tempo, através da coleta continuada de dados e da análise e bloqueio de possíveis causas especiais, responsáveis pelas instabilidades do processo em estudo.

Segundo Borges (2009), a variabilidade indesejada é a grande inimiga da qualidade. Ela está presente em todo processo produtivo, não podendo ser totalmente eliminada, mas mitigada a um nível tolerável. A medição da variabilidade torna-se um indicador efetivo da qualidade do processo produtivo e do produto fabricado.

A visualização simples e eficiente do desempenho e estabilidade, bem como todas as variações de um processo no decorrer do tempo, pode se dar por meio de gráficos de controle - uma das sete ferramentas do CEP (ALENCAR et al., 2005).

Montigomery (2004) cita cinco razões da importância dos gráficos de controle:

1. São uma técnica que reduz o retrabalho. Desta forma, a produtividade aumenta com consequente redução do custo e crescimento da capacidade produtiva.

2. São eficazes na prevenção de defeitos, ajudando a manter o processo sob controle. Como o processo de inspeção é oneroso, deve-se fazer certo da primeira vez. 
3. Evitam o ajuste desnecessário do processo. Um gráfico de controle pode distinguir entre uma causa especial de variação e uma causa aleatória, de modo que nenhum instrumento, incluindo o operador humano é tão eficiente nessa distinção.

4. Fornecem informações de diagnóstico. O padrão de pontos detectado contem informações que serão de grande relevância para mudanças que melhorem o desempenho do processo produtivo.

5. Os gráficos de controle fornecem informações sobre a capacidade do processo.

Resumidamente, esses gráficos de controle, também chamados de cartas de controle de Shewhart, são as ferramentas mais importantes do CEP, pois elas monitoram o processo produtivo determinando se o mesmo se encontra no planejamento ou se determinadas causas não aleatórias estão atuando, detectando desvios de parâmetros representativos do processo, reduzindo a quantidade de produtos fora de especificações e os custos de produção.

A grande contribuição dessa técnica estatística que monitora e rastreia toda a variabilidade existente dentro de um processo de produção é a identificação da fonte e da natureza das causas de variação, trazendo informações importantes para o controle do processo e sua consequente evolução, redundando em conhecimento indispensável aos gestores, é facultar a possibilidade da busca pela qualidade de uma forma sistêmica, ou seja, desde os insumos até o produto final.
As cartas de controle são gráficos temporais que apresentam os valores de medição da variável de interesse no eixo das ordenadas e os pontos no tempo nos quais as medições são efetuadas no eixo das abcissas. Os pontos plotados nos gráficos de controle são unidos por segmentos de reta, que são interpretados em função de linhas horizontais, chamados de limite superior de controle (LSC), linha média (LM) e limite inferior de controle (LIC). Nas pesquisas, realizadas por Shewhart (1931), foi demonstrado que os limites são calculados por meios de recursos matemáticos e são fundamentados na teoria estatística. Quando se encontra um ponto do gráfico acima do LSC ou abaixo do LIC a conclusão é a de que, possivelmente, existe uma causa especial de variação, proporcionando instabilidade ao processo, e caso tal causa seja encontrada, na maioria dos casos, é possível agir pontualmente para eliminá-la. Quando todos os pontos se encontram dentro dos limites de controle, outros padrões de não-aleatoriedade devem ser investigados. Caso nenhum padrão de nãoaleatoriedade seja detectado, a conclusão é de que o processo esta sob a ação de causas comuns, ou seja, está sob controle estatístico.

Lima (2006) citando ASFQC (1986) e Thompson e Koronacki (1993) demonstrou que a variabilidade do processo pode ser monitorada tanto através do gráfico de controle para desvio padrão, (gráfico $S$ ) como para o gráfico de amplitude (gráfico $R$ ). Aconselha-se o uso do gráfico $\mathrm{S}$ quando o 
tamanho dos subgrupos racionais for grande (maior que 30) e o gráfico $R$, caso contrário. Essa recomendação se deve ao fato do estimador do desvio-padrão (S) ser viesado, mas assintoticamente não-tendencioso.

\section{ABATE E RESFRIAMENTO DE AVES}

O frigorífico (unidade industrial ou abatedouro) é o quinto elo da cadeia produtiva onde se origina o produto final - o frango, resfriado, congelado, inteiro e em cortes. É composto na sua maioria por várias seções no processo produtivo, ou seja: recepção e abate; escaldagem e depenagem; evisceração; resfriamento e embalagem; corte e congelados.

Segundo Bueno (2006), a cadeia produtiva da avicultura de corte é, provavelmente, uma das cadeias produtivas brasileiras com maior nível de coordenação, conferindo-lhe grande competitividade no mercado mundial. Com o crescimento do consumo de carne de frango nos últimos anos e devido a crise no setor referente a Influenza aviária, em 2004, que afetou a produção na Ásia, onde redundou na perda de confiança no mundo inteiro, o consumidor está mais exigente quanto à qualidade e a segurança do produto. Sendo o frigorífico o agente coordenador dessa cadeia cabe a ele a responsabilidade quanto à gestão da qualidade voltada às necessidades do mercado consumidor. As crescentes exigências do mercado externo, no que se refere a importação de carne de frango do Brasil, contribuem de maneira decisiva para que o país torne-se um dos melhores produtores de carne de frango do mundo em qualidade e lucratividade. Em 2005, dos 2,4 milhões de toneladas exportadas, aproximadamente 1,5 milhão de toneladas foram de frango em cortes, segmento de maior valor agregado, principalmente para países como Japão, Países Baixos e Alemanha.

De acordo com a UBA (2005), em 2005 as exportações brasileiras foram impulsionadas pelos principais compradores como o Oriente Médio, a Ásia, União Europeia, Rússia, África e o Mercosul, superando a exportação dos EUA.

As exigências legais para a industrialização e processamento dos frangos nos frigoríficos constam à legislação vigente, a portaria $\mathrm{n}^{\mathrm{o}} .210$ de 10 de novembro de 1998 , da Secretaria de Defesa Agropecuária (SDA)/ Ministério da Agricultura Pecuária e Abastecimento (MAPA), prevê a refrigeração e manutenção da temperatura entre $0^{\circ} \mathrm{C}$ (zero grau centígrado) a $4^{\circ} \mathrm{C}$ dos produtos de aves (carcaças, cortes ou recortes, miúdos e/ou derivados), com tolerância de $1^{\circ} \mathrm{C}$ medidos na intimidade dos mesmos (BRASIL, 1998).

De acordo com Dorr e Marques (2004), o futuro do comércio da carne de frango depende fundamentalmente da indústria quanto à garantia da qualidade e da flexibilidade para mudanças. Para tanto as empresas devem executar as atividades de abate e processamento com garantia de 
qualidade. A gestão da qualidade adequada às exigências cada vez maiores do mercado torna-se relevante para os frigoríficos, caso o Brasil pretenda continuar na liderança de exportações de carne de frango. Segundo a UBA (2005) a avicultura de corte brasileira fará todos os esforços juntamente com os órgãos governamentais para que o frango brasileiro seja cada vez mais reconhecido e continue na mesa dos consumidores de todo o mundo.

\section{OBJETIVO}

O objetivo do presente trabalho foi realizar um estudo de caso em uma indústria de abate e resfriamento de aves sul mineira, averiguando a temperatura no interior da câmara fria e inferir se o processo de resfriamento se encontrava sobre controle estatístico de processo.

\section{MATERIAL E MÉTODOS}

Este trabalho possui como objeto de estudo uma indústria de médio porte de abate e resfriamento de aves situada no município de Alfenas, sul do Estado de Minas Gerais, cuja produção se destina somente para ao mercado interno.

$\mathrm{Na}$ coleta dos dados foram utilizadas duas técnicas: a primeira foi uma entrevista com funcionários e gerência, com o objetivo de verificar se alguma técnica de monitoramento da qualidade era adotada pela empresa e como se dava a gestão da qualidade; a segunda foi a verificação das temperaturas na câmara de resfriamento de frangos nos meses de janeiro e fevereiro de 2011, durante trinta e cinco dias ininterruptos $(n=35)$, oito vezes ao dia $(m=8)$.

Para anotações foi criada uma planilha de registro para controle da temperatura durante o período de coleta dos dados. Um funcionário voluntário foi treinado para seu preenchimento.

Para monitorar a variável temperatura, foram utilizados os gráficos de controle para média $\bar{X}$ e para amplitude $R$ - e não gráfico $\mathrm{S}$, pois o tamanho dos subgrupos racionais foi pequeno $(\mathrm{m}=8)$.

As cartas da média e amplitude são usadas quando múltiplas medições das características do processo são coletadas dentro de curto período de tempo, sob as mesmas condições.

A carta $\bar{X}$ exibe a média do atributo do processo, refletindo mudanças que ocorrem no sistema de causas comuns de variação, os quais afetam o desempenho do processo de uma forma geral.

A correspondente carta $R$ indica a dispersão existente dentro dos subgrupos, sendo sensível a mudanças que afetam somente parte do processo.

As equações para determinar a linha média (LM) e os limites de controle superior (LSC) e inferior (LIC) das cartas para média e amplitude são dadas a seguir na tabela abaixo (Montgomery, 1996). 
Tabela 1 - Limites de controle das cartas $\bar{X}$ e $R$.

\section{Limites de Controle}

\begin{tabular}{cl}
\hline Carta da Média $(\bar{X})$ & Carta da Amplitude $(R)$ \\
\hline$L S C_{\bar{x}}=\overline{\bar{X}}+A_{2} \bar{R}$ & $L S C_{R}=D_{4} \bar{R}$ \\
\hline$L M_{\bar{x}}=\overline{\bar{X}}$ & $L M_{R}=\bar{R}$ \\
\hline$L I C_{\bar{x}}=\overline{\bar{X}}-A_{2} \bar{R}$ & $L I C_{R}=D_{3} \bar{R}$
\end{tabular}

Fonte: Montgomery (1996).

Os termos $A_{2}, D_{3}$ e $D_{4}$, na Tabela 1 são valores tabelados (FERREIRA e OLIVEIRA, 2008), representados por símbolos de fatores para conversão de médias de subgrupos em estimativas não-viesadas (isto é, não-tendenciosas) para limites 3 sigma. Além disso, $\overline{\bar{X}}$ é a média das médias em cada tempo e $\bar{R}$ é a amplitude média.

Outra técnica utilizada foram os índices de capacidade do processo $\left(\mathrm{C}_{\mathrm{p}}\right.$ e $\left.\mathrm{C}_{\mathrm{pk}}\right)$, associados a histogramas amostrais.

De acordo Ferreira e Oliveira (2008), a capacidade do processo é definida a partir de uma comparação dos limites de especificação, os quais expressam as especificações esperadas para o processo. São eles, o Limite Inferior de Especificação (LIE), o Limite Superior de Especificação (LSE) e o Limite Central de Especificação (LCE).

Quanto maior o valor de $C_{p}$, maior a capacidade do processo, sendo que um valor de $C_{p}>1$ indica que o processo é capaz de atender às especificações do cliente.

O cálculo do índice de capacidade de processo $\mathrm{C}_{\mathrm{p}}$, em função $\bar{R}$, é dado por:

$$
C_{p}=\frac{L S E-L I E}{6 \frac{\bar{R}}{d_{2}}} .
$$

A definição do índice $C_{p}$ pressupõe que o processo está centrado no valor nominal da especificação; se este não é o caso, a capacidade real do processo é menor do que a indicada por $\mathrm{C}_{\mathrm{p}}$. Em tal situação, convém a utilização do índice $\mathrm{C}_{\mathrm{pk}}$, para o efeito de distribuição não centrada. $O$ índice $C_{p k}$ avalia a distância do valor central do processo aos limites da especificação, tomando aquela que é menor e, portanto, mais sujeita a propiciar resultados fora de especificação. O cálculo do índice $\mathrm{C}_{\mathrm{pk}}$ é dado por:

$$
\begin{gathered}
C_{p i}=\frac{\bar{X}-L I E}{3 \frac{\bar{R}}{d_{2}}} \quad C_{p s}=\frac{L S E-\bar{X}}{3 \frac{\bar{R}}{d_{2}}} \\
C_{p k}=\min \left(C_{p i}, C_{p s}\right)
\end{gathered}
$$

em que $\mathrm{C}_{\mathrm{pi}}$ é a capacidade inferior e $\mathrm{C}_{\mathrm{ps}}$ é a capacidade superior.

Todas as análises e gráficos foram feitos no pacote CEPpt (BASTOS, 2011) do software estatístico R (R DEVELOPMENT CORE TEAM, 2011). 
$\mathrm{Na}$ análise da temperatura por meio do par de cartas de controle $\bar{X}-R$, pode-se observar a enorme oscilação ao longo do tempo, com muitas médias e amplitudes médias estando fora dos limites de controle (figuras 1a e 1b). Tal fato já é suficiente para afirmar que o processo de resfriamento se encontra fora de controle - não sendo necessário investigar outros padrões de nãoaleatoriedade e indicando que existem causas

\section{RESULTADOS E DISCUSSÃO}

Após a entrevista feita na agroindústria, pode-se constatar que a mesma não possui nenhum programa formalmente implantado de controle da qualidade. As metas que a referida indústria possui no tocante à qualidade são verificadas apenas ao final do processo produtivo, por meio de inspeção. Além disso, ficou explícito que a temperatura de resfriamento em que trabalham as câmaras frias, não é monitorada.

Entretanto, o não monitoramento da temperatura de resfriamento pode trazer danos não apenas à qualidade físico-química e microbiológica do produto final, mas também gerar desconformidades com a legislação vigente e ocasionar autuações junto aos órgãos de inspeção, como a vigilância sanitária e o Ministério da Agricultura. especiais gerando variabilidade. Na Figura 1c pode-se notar que boa parte dos dados se encontra fora dos limites especificados pela legislação $\left(0^{\circ} \mathrm{C}\right.$ a $\left.4^{\circ} \mathrm{C}\right)$ e que o histograma se distancia muito de uma distribuição Normal (que era esperada pelo Teorema Central do Limite). Além disso, o índice $\mathrm{C}_{\mathrm{pk}}$ foi igual a 0,55 , indicando a incapacidade do processo de atender ao especificado. Analisou-se apenas o $\mathrm{C}_{\mathrm{pk}}$ porque o processo não estava centrado.

$\mathrm{Na}$ análise da temperatura por meio do par de cartas de controle $\bar{X}-R$, pode-se observar a enorme oscilação ao longo do tempo, com muitas médias e amplitudes médias estando fora dos limites de controle (figuras 1a e 1b). Tal fato já é suficiente para afirmar que o processo de resfriamento se encontra fora de controle - não sendo necessário investigar outros padrões de nãoaleatoriedade e indicando que existem causas especiais gerando variabilidade. Na Figura 1c pode-se notar que boa parte dos dados se encontra fora dos limites especificados pela legislação $\left(0^{\circ} \mathrm{C}\right.$ a $\left.4^{\circ} \mathrm{C}\right)$ e que o histograma se distancia muito de uma distribuição Normal (que era esperada pelo Teorema Central do Limite). Além disso, o índice $\mathrm{C}_{\mathrm{pk}}$ foi igual a 0,55 , indicando a incapacidade do processo de atender ao especificado. Analisou-se apenas o $\mathrm{C}_{\mathrm{pk}}$ porque o processo não estava centrado. 


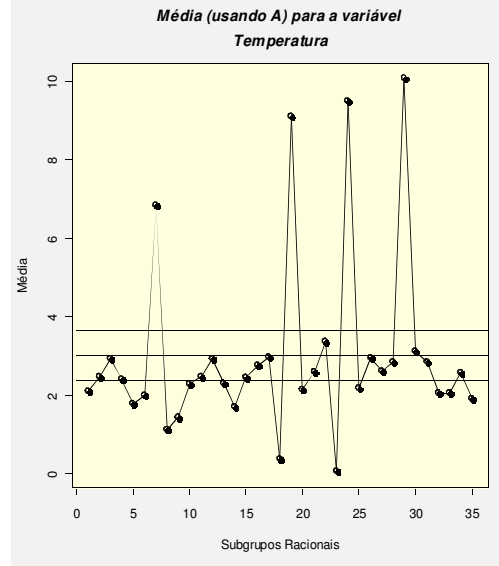

(a)

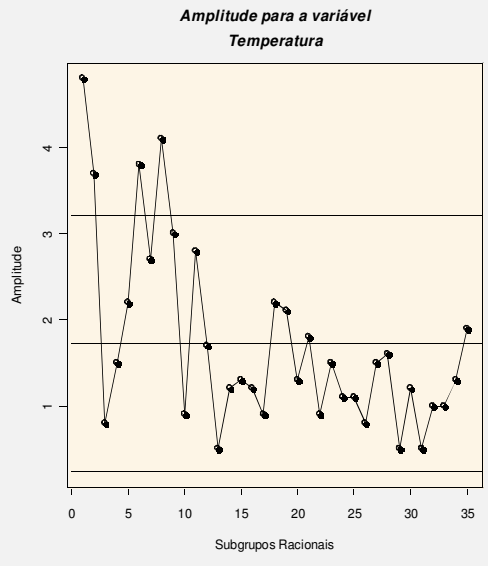

(b)

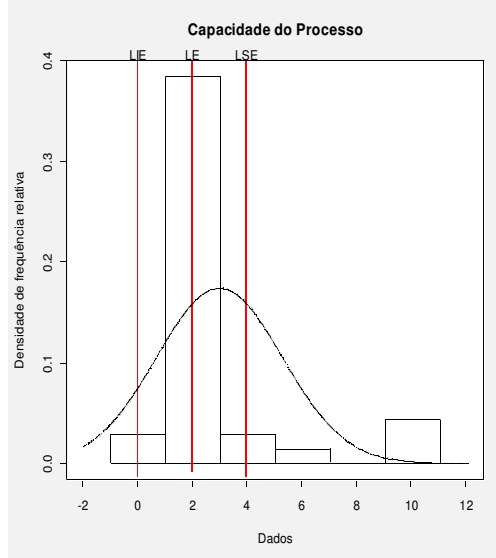

(c)

Figura 1 Cartas de controle $\bar{X}$ (a), R (b) e capacidade do processo (c) para a temperatura (primeira análise).

Após essa evidência da existência de causas especiais de variabilidade, voltou-se à indústria e foi verificado que em alguns dos dias informados a câmara fria esteve vazia, gerando dados inúteis para análise. Diante disso, esses dados foram descartados e novas análises foram feitas.

$\mathrm{Na}$ Figura 2a nota-se que, após a retirada dos dados inadequados, as médias do processo se mostraram muito melhor comportadas mas, mesmo assim, apresentando pontos fora de controle. A variabilidade se manteve semelhante, ainda apresentando desconformidades (Figura 2b). Pela Figura 2c nota-se que praticamente todos os dados se encontram dentro dos limites de especificação e o histograma se assemelha muito mais à distribuição Normal. Além disso, o novo $C_{p k}$ foi igual a 0,95 e o $C_{p}$ igual a 1,09 , mostrando que o processo pode ser capaz de atender às especificações e que sua média é mais similar à temperatura média especificada do que se imaginava.

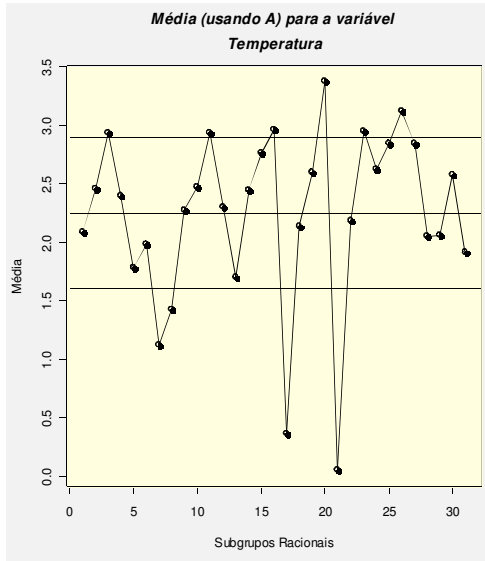

(a)

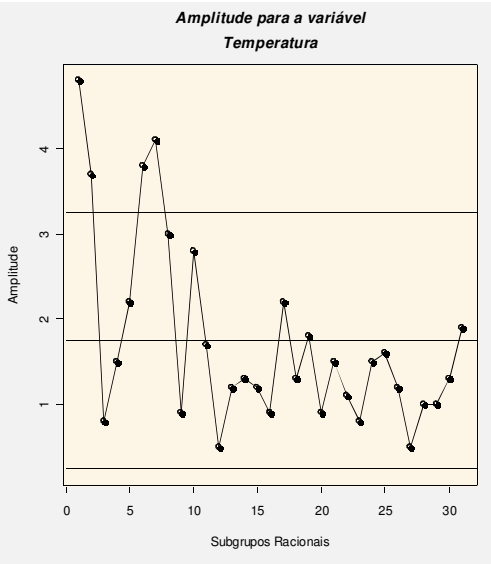

(b)

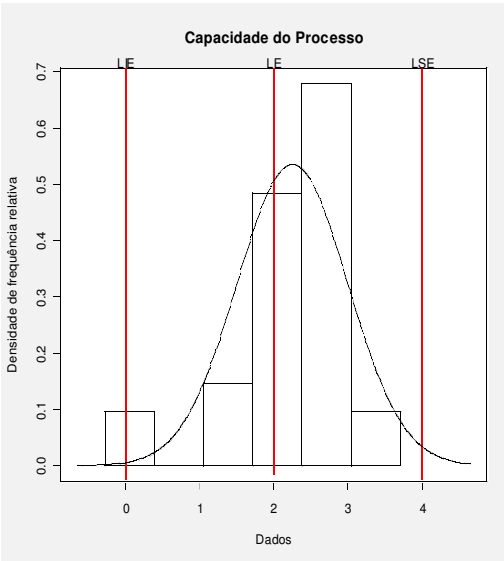

(c)

Figura 2 Cartas de controle $\bar{X}$ (a), R (b) e capacidade do processo (c) para a temperatura (segunda análise). 


\section{CONCLUSÕES}

Perante os resultados das análises conclui-se que o processo de resfriamento de aves da indústria em foco não está sob controle. Mesmo removendo-se os dias em que a câmara trabalhou vazia, existem outras fontes detectáveis gerando variabilidade indesejada e o processo pode ser melhorado para que atenda às exigências fitossanitárias e de segurança alimentar.

\section{REFERÊNCIAS}

ALENCAR, J. R. B. Validação do processo de fabricação de formasfarmacêuticas sólidas usando CEP e redes neurais. [Examede qualificação] Rio de Janeiro: Centro de Tecnologia, Escolade Química, Universidade Federal do Rio de Janeiro; 2004.

ALENCAR, J. R. B.; SOUZA JÚNIOR, M. B.; ROLIM NETO, P. J.; LOPES, C. E. Uso de controle estatístico de processo para avaliação da estabilidade e validação da fase de compressão de formas farmacêuticas sólidas. Acta Farm Bonaer. 24(3):426-35. 2005.

ASFQC. American Society for quality control. Statistical process control manual. Milwaukee, ASQC, 1986.

BASTOS, R. L. CEPpt: um pacote R para o Controle Estatístico de Processo. 2011. 36p. Trabalho de Conclusão de Curso (Graduação em Matemática). Universidade Federal de Alfenas, Alfenas, 2011.

BORGES, R. C. Estudo de testes de estabilidade de processos em gráficos de controle de Shewhart, 2009. 144p.

Dissertação (Mestrado em Estatística e Experimentação Agropecuária) Universidade Federal de Lavras, Lavras-MG.
BRASIL. Ministério da Agricultura Pecuária e Abastecimento (MAPA). Regulamento Técnico da Inspeção Tecnológica e HigênicoSanitária de Carnes de Aves. Diário Oficial da União, Brasília, DF. 1998.

BUENO, M. P. Gestão da qualidade nos frigoríficos de abate e processamento de frangos no estado de Mato Grosso do Sul. 85f. Dissertação (Mestrado em Agronegócios) - Universidade Federal de Mato Grosso do Sul. Campo Grande: UFMS. 2006.

CORTIVO, Z. D. Aplicação do Controle Estatístico de Processoem seqüências curtas de produção e análise estatística deprocesso através do planejamento econômico.

[Dissertação] Curitiba: Universidade Federal do Paraná; 2005.

DORR, A.; MARQUES, P.V. Respostas às exigências: matérias-primas. Rev. Avicultura Industrial. Edição 1122, n.4, 2004.

FERREIRA, E. B.; OLIVEIRA, M. S. de. Controle estatístico da qualidade. Lavras: UFLA/FAEPE, 2008. 87p.

JURAN, J. M.; GRYNA, F. M. Controle da qualidade: métodos estatísticos clássicos aplicados à qualidade. 4th ed. São Paulo: Makron, v. 6. 1993.

LIMA, A. A. N.; LIMA, J. R.; SILVA, J. L.; ALENCAR, J. R. B.; SOARES-SOBRINHO, J. L.; LIMA, L. G.; ROLIM-NETO, P. J. Aplicação do controle estatístico de processo na indústria farmacêutica. Revista Ciências Farmacêuticas Básica Aplicada., v. 27, n.3, p.177-187, 2006.

MONTEGOMERY, D. C. Introdução ao controle estatístico da qualidade. 4. ed. Rio de Janeiro: LTC, 2004. 516 p.

PARANTHAMAN, D. Controle de qualidade. São Paulo: McGraw- Hill Ltda. p.118-212. 1990.

R DEVELOPMENT CORE TEAM. $R: A$ language and environment for statistical 
computing. R Foundation for Statistical Computing, Vienna, Austria. 2011.

SHEWHART, W. A. Economic control of quality of manufactured product. New York: Van Nostrand, 1931. 501 p.

SILVA, L. S. C. V. Aplicação do controle estatístico de processos na indústria de laticínios Lacatoplasa: um estudo de caso. [Dissertação]. Florianópolis: Universidade Federal de Santa Catarina; 1999.

UBA, União Brasileira de Avicultura. Relatório anual 2004/2005. 2005. Disponível em: <http://www.uba.org.br $>$. Acessado em: 31 ago 2010. 